\title{
Effect of vitrification procedure on chromosomal status of embryos achieved from vitrified and fresh oocytes
}

\author{
Javier Israel García*, Luis Noriega-Portella, Luis Noriega-Hoces \\ Laboratory of Assisted Reproduction, PRANOR Group of Assisted Reproduction, Concebir Clinic, Lima, Peru. \\ *Corresponding Author: igarciaf@hotmail.com
}

Received 27 April 2011; revised 9 June 2011; accepted 28 June 2011.

\begin{abstract}
background: In order to assess the chromosomal status in embryos obtained from vitrified and fresh donated oocytes, preimplantational genetic diagnostic (PGD) was performed after biopsy of one blastomere at day 3. METHODS: A total of 249 oocytes were obtained from 23 oocyte donors, 80 oocytes were used in the vitrified group and 151 oocytes were used in the fresh group. Nine chromosomes $(13,15,16,17$, $18,21,22, X$ and $Y$ ) were investigated by fluorescence in situ hybridization (FISH) analysis in 56 and 121 embryos from vitrified and fresh group respectively. Fertilization, cleavage rate, embryo quality and chromosomal abnormality rate were compared between evaluated groups. Results: Vitrified oocytes showed a survival rate of $97.5 \%$. There was no significant difference in the fertilization rate $(82.7 \%$ and $91.4 \%)$, Day 2 cleavage rate $(90.3 \%$ and $87.7 \%)$ or blastocyst formation rate $(31.1 \%$ and $44.6 \%)$ for the vitrified and fresh groups respectively. Chromosomal abnormality rate $(66.1 \%$ versus $71.9 \%)$, percentage of abnormal blastocysts $(61.1 \%$ versus $64.8 \%$ ) and percentage of abnormalities for each analyzed chromosome were similar for the vitrified group compared with the control group. Conclusions: The rates of chromosomal abnormalities in embryos from vitrified oocytes are similar to those published previously; and comparable to those observed in embryos from fresh oocytes. These results confirm that the developmental competence and chromosomal status of embryos obtained from vitrified oocytes is not affected by the vitrification procedure, and they preserve the potential to be fertilized and to develop in to blastocyst stage similar to embryos from fresh oocytes.
\end{abstract}

Keywords: Oocytes; Vitrification; PGD; FISH; ART

\section{INTRODUCTION}

Human oocytes cryopreservation is an attractive choice to the range of fertility treatments presently offered. Since the very first frozen oocyte pregnancy was achieved in humans by Chen [1], the slow cooling method has been applied with varying success [2-7]. There are many reports about the cryopreservation of human oocytes with good fertilization and cleavage rates $[1,8]$ but with variable results on pregnancy rates per cycle [9-13]. A recent meta-analysis on slow freezing revealed that the clinical pregnancy rate per transfer with this method was $20.6 \%$, and $2.3 \%$ per thawed oocyte [14].

As an alternative to slow freezing, the vitrification procedure has been recently applied for human oocytes and embryos $[15,16]$ allowing to improve the percentages of oocyte survival, fertilization, embryo development rates and clinical outcomes [17-26]. Vitrification is a non-equilibrium method and may be regarded as a radical approach in which ice crystal formation is totally eliminated. Nevertheless, it requires an extremely high cooling rates and high concentrations of cryoprotectants, compared to slow freezing [16], in which a solution/ specimen solidifies to form a glass-like, or vitreous, structure without any ice crystal formation during cooling. This state is maintained throughout the whole warming procedure [22].

The difficulties associated with oocyte cryopreservation are mostly related to the special structure and sensitivity of this cell, the exposure time to the cryoprotectants solutions, the concentration of the cryoprotectants, the equilibrium temperature and the extra and intracellular ice formation factors that affect the viability [10] and physiology of oocytes [27]. Additionally, other biological characteristics of human oocytes that may be susceptible to damage during the cryopreservation procedure are precocious oocyte activation induced by cryoprotectants exposures that may disturb future development 
[27,28]; loss of high mitochondrial polarity associated with a significantly reduced capacity to up-regulate the levels of intracellular free calcium after thawing [29]; microvacuolization in the ooplasm and ultrastructural alterations in specific oocyte microdomains linked to the reduced developmental potential of mature cryopreserved oocytes [30].

Proper organization of the oocyte cytoskeleton, particularly of its microtubular fraction, is essential for normal spindle formation and chromosome segregation [30]. The meiotic spindles are crucial for the events following fertilization in the completion of meiosis, second polar body formation, migration of pronuclei, and formation of the first mitotic spindle [31]. The meiotic spindle has been shown to be extremely sensitive to temperature variation [32-41], and to depolymerize at low temperatures $[42,43]$. Subsequent impaired repolymerization on rewarming may lead to scattering of chromosomes or lesions in the reformed spindle, resulting in misaggregation of chromatids following resumption of meiosis and an increase of aneuploidies incidence in oocytes [44] and later in embryos [45,46].

On the other hand, several studies evaluating the effect of vitrification and slow-freezing on the meiotic spindle integrity and chromosome alignment [47-51], showed less damage in vitrified oocytes (as observed in fresh oocytes without cryopreservation) compared with cryopreserved oocytes by slow-freezing. However, studies of Coticchio et al. [52]; Martinez-Burgos et al. [53] showed that the vitrified-warmed oocytes maintain a metaphase II (MII) spindle with a bipolar organization, but the chromosomal alignment appears to be partly compromised. Additionally, studies of Cobo et al. [19], Chen and Yang, [36] and Ciotti et al. [54] reported that spindle recovery was faster in vitrification than in slow freezing (1-2 hours after thaw procedure). However, most of the available information about of chromosomal status comes from studies performed on cryopreserved oocytes, but not on the embryos derived from those oocytes.

The introduction of preimplantation genetic diagnosis (PGD) to the field of reproductive medicine and the analysis of a single blastomere from a day 3 embryo using multicolor fluorescence in-situ hybridization (FISH), permit to detect chromosomal abnormalities and inherited diseases without adversely affecting the developmental [55] or implantation potential of the embryo [56,57]. Routinely, up to 9 to 12 chromosomes can be analyzed on a single fixed nucleus by FISH, in which DNA probes labeled with fluorochromes hybridize to their respective chromosome, allowing for the identification of ploidy status. The indications for PGD during an IVF cycle are advanced maternal age, repeated implanttation failure, recurrent pregnancy loss and, previous trisomic conception [58].

The relationship between chromosome abnormalities, embryo morphology and maternal age has been studied extensively [50-62]. Approximately 30\% of human embryos generated from IVF treatments have an abnormal chromosome constitution $[63,64]$, and this percentage may increase to $60 \%-70 \%$ in embryos that come from poor prognosis IVF patients such as low responders, older women [65-67], IVF failure [56,68,69], or women with a history of recurrent miscarriage [63,69-73]. In a recent study, involving an examination of nine chromosomes at the cleavage stage via FISH, $60 \%$ of all analyzed embryos derived from women younger than 35 years were found to carry cytogenetic anomalies. The observed abnormality rate increased to $80 \%$ for embryos of women 41 years of age and older [74].

On the other hand, young egg donors are traditionally assumed to have low rates chromosome abnormalities, and young donors less than infertile patients, even those with comparable ages. The scanty published PGD data on embryos derived from oocytes donors indicate unexpectedly high rates of chromosome anomalies $(56 \%$ $57 \%$ ) [50,75]. According to Reis Soares et al. [75] the reason for the observed abnormalities, could be that donors are frequently subject to more aggressive stimulation, compared with other women of similar age, in order to guarantee the production of a large cohort of oocytes.

Nevertheless, the effect of cryopreservation procedures on chromosomal status of embryos achieved from cryopreserved oocytes has been little studied. Cobo et al. [76] analyzed the effect the cryopreservation by slow freezing on the chromosomal status of embryos obtained from frozen-thawed oocytes. They showed $28.6 \%$ and $26 \%$ of aneuploidies in embryos obtained from cryopreserved and fresh oocytes respectively.

This study was designed to compare the rates of chromosome abnormalities observed in embryos obtained from vitrified and fresh donated oocytes and to evaluate whether the vitrification procedure increase significantly more chromosome abnormalities.

\section{MATERIALS AND METHODS}

\subsection{Patients}

This study is based on secondary analysis of data obtained from procedure of Preimplantational Genetic Diagnostic (PGD) program in the Laboratory of Assisted Reproduction at the Concebir Clinic (Lima, Peru) carried out over 2009. This study was approved by the Institutional Review Board (IRB) and the corresponding Ethics Committee. Written informed consent forms were obtained from donors, recipients and their partners included in this study. The PGD indications were because 
all patients wish to avoid a pregnancy with trisomic disease.

The age of the oocyte donors ranged from 20 - 29-year-old in both evaluated groups. They were subjected a physical, gynecological and psychological examination and there were no family history of hereditary or chromosomal diseases. All participants had a normal karyotype and tested negative in a screening for sexually transmitted diseases.

From the 23 oocyte donors included, 8 were assigned to the vitrified oocytes group and; 15 , to the fresh oocytes group. A total of 82 and 167 oocytes were obtained from vitrified and fresh oocytes groups respectively.

Recruitment of oocyte donors was done based on recommendations given by other donors and the donation of their gametes was merely by altruistic reasons. The donors and their oocytes were random and consecutively assigned each evaluated group. They were matched with their recipients according to phenotype and blood groups; and the recipients were not aware not of the origin of oocyte received (vitrified or fresh).

\subsection{Controlled Ovarian Stimulation and Oocyte Collection}

The menstrual cycles of oocyte donors were stimulated using recombinant FSH (rFSH) (Puregon ${ }^{\circledR}$, Organon laboratories, Peru) and GnRH antagonist (Orgalutran ${ }^{\circledR}$ Organon Laboratories, Peru) according to the stimulation protocols previously established and starting on Day 2 of the menstrual cycle until when at least three follicles reached $\sim 18 \mathrm{~mm}$ in diameter. The oocyte retrieval was performed by vaginal ultrasound $36 \mathrm{~h}$ after the i.m application of Human Chorionic Gonadotropin, hCG (Ovidrel ${ }^{\circledR} 250 \mu \mathrm{g}$, Serono Laboratories, Peru). For the procedure, the patient was under general anesthesia with $200 \mathrm{mg}$ of Propofol iv (Diprivan ${ }^{\circledR}$ 1\% P/V; AstraZeneca Laboratories, UK).

During follicular aspiration procedure the oocytes were recovered in Global®-HEPES-buffered medium (IVFonline, Canada) supplemented with $10 \% \mathrm{vol} / \mathrm{vol}$ Serum Substitute Supplement (SSS; Irvine Scientific, USA). After retrieval, cumulus-oocyte complexes were trimmed of excess cumulus cells and maintained in $\sim 200$ $\mu \mathrm{L}$ drops of Global ${ }^{\circledR}$-Fertilization medium (IVFonline, Canada) plus $10 \% \mathrm{SSS}$ under oil at $37^{\circ} \mathrm{C}$ and an atmosphere containing $5.6 \% \mathrm{CO}_{2}, 5 \% \mathrm{O}_{2}$ and $89.4 \% \mathrm{~N}_{2}$, for 2 hours until when the oocytes recovered were cryopreserved (vitrified group) or 5 hours until when the oocytes recovered were inseminated (fresh group).

All collected oocytes were denuded enzymatically of cumulus cells with hyaluronidase $(80 \mathrm{IU} / \mathrm{mL}$; IVFonline, Canada) to assess nuclear maturity. Only metaphase II (MII) oocytes were vitrified with Cryotop minimum volume vitrification methods [21,48,77] using comercial cryoprotectant solutions (Cecolfes, Colombia) and the Cryolock device (Biodiseño-Colombia), for a maximum period of 3 hours after of retrieval.

\subsection{Oocyte Vitrification}

A total of 80 MII oocytes were vitrified which corresponding to the vitrified group by minimum volume methods, which minimize the volume of vitrification solution containing oocytes increasing the cooling and warming rates and decreasing the chance of ice crystal nucleation/formation in the small sample [16,78]. The vitrification procedure was performed at room temperature $\left(24^{\circ} \mathrm{C}\right)$. The oocytes were equilibrated in $7.5 \%$ vol/vol ethylene glycol (EG) plus 7.5\% dimethylsulfoxide (DMSO) in TCM199 medium for 15 minutes (equilibrium solution, ES) and then placed in $15 \%$ EG plus $15 \%$ DMSO plus $0.5 \mathrm{mmol} / \mathrm{L}$ sucrose (vitrification solution, VS) for a maximum 1 minute. The oocytes were loaded quickly onto the Cryolock in a minimum drop size of $<0.1 \mu \mathrm{L}$ of VS and immediately immersed directly in liquid nitrogen, at a cooling rate of approximately $-23,000^{\circ} \mathrm{C} / \mathrm{min}$ [21]. A maximum three oocytes were placed by Cryolock. All cryoprotectant solutions were supplemented with $20 \% \mathrm{vol} / \mathrm{vol}$ SSS.

\subsection{Oocyte Warming}

Oocytes were warmed at fast warming rates of $-12,000^{\circ} \mathrm{C} / \mathrm{min}$ [21]. The Cryolock was taken out of liquid nitrogen and quickly placed in $1.0 \mathrm{~mol} / \mathrm{L}$ sucrose in TCM199 medium (thawing solution-TS) for 1 minute at $37^{\circ} \mathrm{C}$. The oocytes were then placed in $0.5 \mathrm{~mol} / \mathrm{l} \mathrm{su}-$ crose in TCM199 medium (diluent solution-DS) for 3 minutes and at room temperature $\left(24^{\circ} \mathrm{C}\right)$, followed by two consecutive 5-min flushes in TCM199 medium (washing solution-WS). Oocytes were then immediately placed in Global ${ }^{\circledR}$-Fertilization medium plus $10 \%$ SSS under oil at $37^{\circ} \mathrm{C}$ and an atmosphere containing 5.6\% $\mathrm{CO}_{2}$, $5 \% \mathrm{O}_{2}$ and $89.4 \% \mathrm{~N}_{2}$ for 2 hours before ICSI. All warm solutions were supplemented with $20 \%$ vol/vol SSS.

\subsection{Insemination, Fertilization and Embryo Culture}

In the vitrified group, $2 \mathrm{~h}$ after warming the viability of oocytes was evaluated microscopically based on the morphology of the oocyte membrane integrity. All viable oocytes were inseminated by intracytoplasmic sperm injection (ICSI) with spermatozoa from recipient's husband. After ICSI procedure (Day 0), all injected oocytes were cultures at $37^{\circ} \mathrm{C}$ in an atmosphere of $5.6 \% \mathrm{CO}_{2}$, $5 \% \mathrm{O}_{2}$ and $89.4 \% \mathrm{~N}_{2}$.

In the fresh group, $\sim 5 \mathrm{~h}$ after of oocyte retrieval, all 
MII oocytes were inseminated or injected, depending about seminal characteristics, with spermatozoa from recipient's husband.

In both groups, the fertilization was evaluated $16-18$ hours after insemination by presence of two pronuclei (Day 1). The zygotes were individually cultured under mineral oil, in $10 \mu \mathrm{L}$ droplets of Global ${ }^{\circledR}$ medium (IVFonline, Canada) supplemented with $10 \%$ vol $/$ vol SSS from Day 1 to Day 3. On Day 3, the embryos were biopsied when they had seven-eight blastomeres. After embryo biopsy, the embryos were moved to fresh $10 \mu \mathrm{l}$ droplets of Global ${ }^{\circledR}$ medium $+10 \%$ SSS.

On Day 2 and 3 the embryos were evaluated for cell number and fragmentation; and on Day 5 for development to blastocyst and expansion.

\subsection{Embryo Biopsy, Fixation, and FISH Analysis}

Embryo biopsies were performed on the third day after insemination, at the $7-8$ blastomeres stage following a protocol described elsewhere [57]. Individual embryos were placed into calcium/magnesium-free media (PGD Biopsy Medium; IVFonline, Canada). The hole of the zone pellucida was opened with Tyrode's acid solution and one nucleated blastomere was removed mechaniccally. Blastomeres were fixed individually following a protocol to minimize signal overlap and loss of micronuclei [79]. After biopsy all biopsied embryos were rinsed thoroughly and individually cultured under oil in $10-\mu 1$ droplets of Global ${ }^{\circledR}$ medium $+10 \%$ SSS at $37^{\circ} \mathrm{C}$ and an atmosphere containing 5.6\% $\mathrm{CO}_{2}, 5 \% \mathrm{O}_{2}$ and $89.4 \% \mathrm{~N}_{2}$.

PGD analysis was performed by FISH using probes specific for nine chromosome types X, Y, 13, 15, 16, 17, 18, 21 and 22. These probes were used because in the past their use has shown improvements in implantation rates after PGD $(56,57)$. The FISH analysis consisted of two consecutive hybridizations following previously published protocols [80]. The first hybridization was performed with probes for chromosomes 13, 16, 18, 21 and 22 (Multivysion PB; Vysis, Downer'Grove, IL, USA). The second hybridization consisted of a homemade combination of probes for chromosomes X, Y, 15 and 17 [80]. Scoring was performed by eye without the need of any software. If the specific signals for a chromosome were not clearly diagnosable, a third hybridization using a probe binding to a different locus for that chromosome was used [81].

\subsection{Embryo Quality}

On Day 2 and 3 the embryos were evaluated for cell number, fragmentation, and multinucleation. Good qual- ity Day 2 embryos were defined as those with 2 - 4 cells and $\leq 10 \%$ of fragmentation and absence of multinucleation. Good-quality Day 3 embryos were defined as those with 6 - 8 cells and $\leq 10 \%$ of fragmentation. Good-quality blastocysts were defined as having an inner cell mass (ICM) and trophoectoderm type A or B [82]. The ICM score was evaluated as follow: type $\mathrm{A}=$ compact area, many cells present; type $\mathrm{B}=$ cells are loosely grouped. The trophoectoderm was scored as follows: type $\mathrm{A}=$ many cells forming a tight epithelial network of cells; type $\mathrm{B}=$ few cells forming a loose network of cells.

\subsection{Sperm Collection}

The semen samples were collected by masturbation from the recipients' partners. Motile spermatozoa were separated from the seminal plasma by centrifugation through $1.0 \mathrm{~mL} \mathrm{95 \%} \mathrm{and} \mathrm{45 \%} \mathrm{Isolate} \mathrm{gradients} \mathrm{(Irvine}$ Scientific, USA). For oligospermic samples the sperm were washed and resuspended in varying amounts of sperm washing medium depending on initial concentration and motility and then placed into $10 \mu \mathrm{L}$ drops of HEPES-buffered Global medium $+10 \%$ SSS for ICSI.

\subsection{Statistical Analysis}

Data were statistically analyzed using the $\chi^{2}$ test and Student's t-test as appropriate and differences were considered to be significant at $P<0.05$. All statistical analysis was carried out using the statistic package Stata 10 (StataCorp, College Station, TX, USA).

\section{RESULTS}

The ages of oocyte donors were similar in both evaluated groups ( $24.6 \pm 3.5$ vs. $24.9 \pm 2.7$ years; P:NS). The mean days of stimulation $(9.4 \pm 0.9$ vs. $9.1 \pm 0.8)$, mean days of antag-GnRH $(1.6 \pm 0.7$ vs. $1.5 \pm 0.7)$ and $\mathrm{rFSH}$ treatment $(1825 \pm 291.6$ vs. $1801.7 \pm 261.1 \mathrm{IU} / \mathrm{L})$ were similar between vitrified and fresh groups (data no shown).

Results obtained from vitrified and fresh group are shown in Table 1. A total of 82 and 167 oocytes were collected from donors of vitrified and fresh groups, respectively. In the vitrified group, there were $80 \mathrm{MII}$ oocytes vitrified, resulting in the survival of 78 oocytes $(97.5 \%)$. Seventy five and 151 oocytes from vitrified and fresh groups, respectively, were inseminated. The normal fertilization (2PN) was similar in both evaluated groups (Vitrified group: $82.7 \%$ vs. Fresh group: $91.4 \%$ ). In the vitrified group, $90.3 \%$ of zygotes underwent cleavage on Day 2, whereas for zygotes from fresh group, the cleavage rate was $87.7 \%$. Mean cell numbers at Days 2 and 3 were similar in both groups. Embryo quality from vitrified oocytes was similar to those derived from fresh oo- 
Table 1. Laboratory results in the vitrified and the fresh group.

\begin{tabular}{ccc}
\hline & $\begin{array}{c}\text { Vitrified } \\
\text { group }\end{array}$ & $\begin{array}{c}\text { Fresh } \\
\text { group }\end{array}$ \\
\hline No. total oocytes & 82 & 167 \\
No. total MII oocytes & 80 & 151 \\
No. total vitrified oocytes & 80 & - \\
No. total oocytes survival (\%) & $78(97.5)$ & - \\
No. total injected oocytes & 75 & 151 \\
No. total fertilized oocytes (2PN) (\%) & $62(82.7)$ & $138(91.4)$ \\
Cleavage rate by embryo at Day 2 (\%) & 90.3 & 87.7 \\
No. cell/embryo at Day 2 (Mean \pm SD) & $3.63 \pm 0.40$ & $3.89 \pm 0.46$ \\
Good quality embryos at Day 2 (\%) & 91.1 & 92.6 \\
No. cell/embryo at Day 3 (Mean \pm SD) & $7.11 \pm 1.01$ & $7.43 \pm 0.97$ \\
Good quality embryos at Day 3 (\%) & 80.4 & 86.8 \\
No. total blastocyst (\%) & $18(32.1)$ & $54(44.6)$ \\
Good quality blastocyst (\%) & 66.7 & 75.9 \\
Early blastocyst (\%) & 33.3 & 24.1 \\
Full blastocyst (\%) & 44.4 & 59.3 \\
Expanded blastocyst (\%) & 16.7 & 14.8 \\
Hatching blastocyst (\%) & 5.6 & 1.8 \\
\hline
\end{tabular}

P:NS

cytes on Day 2 (91.1\% vs. 92.6\%) and Day 3 (80.4\% vs. $86.8 \%$ ). Blastocyst development rates were similar (32.1\% and $44.6 \%$, respectively) for the vitrified and the fresh groups. In addition, embryos reaching the blastocyst stage were morphologically similar in both groups.

Table 2 shows the results obtained after FISH analysis. A total of $61(98.4 \%)$ and $129(93.5 \%)$ embryos were biopsied from vitrified and fresh groups respectively. A hundred percent of blastomeres biopsied were fixed in both evaluated groups. $91.8 \%$ and $93.8 \%$ of fixed blastomeres were analyzed for both the vitrified and the fresh groups. The proportion of abnormal embryos obtained from vitrified oocytes was similar to those observed embryos obtained from fresh oocytes (66.1\% vs. $71.9 \%$; respectively). Additionally, from those embryos that reached the blastocyst stage, $61.1 \%$ and $64.8 \%$ in the vitrified and fresh groups respectively, showed chromosomal abnormalities to nine analyzed chromosomes.

The percentage of abnormalities for each chromosome analyzed in both analyzed groups is shown in Table 3 . The proportion of abnormalities according to the different chromosomes analyzed was similar between embryos obtained from vitrified and fresh oocytes.

\section{DISCUSSION}

Since the first pregnancy achieved with a vitrified/warmed human oocyte [83] and the first birth of a healthy baby [84], vitrification results have improved
Table 2. Results of fluorescence in situ hybridization analysis in embryos obtained from vitrified and fresh oocytes.

\begin{tabular}{ccc}
\hline & $\begin{array}{c}\text { Vitrified } \\
\text { group }\end{array}$ & $\begin{array}{c}\text { Fresh } \\
\text { group }\end{array}$ \\
\hline No. total zygotes (2PN) & 62 & 138 \\
No. total biopsied embryos (\%) & $61(98.4)$ & $129(93.5)$ \\
No. total fixed embryos (\%) & $61(100)$ & $129(100)$ \\
No. total analyzed embryos (\%) & $56(91.8)$ & $121(93.8)$ \\
No. total abnormal embryos (\%) & $37(66.1)$ & $87(71.9)$ \\
No. total blastocyst & 18 & 54 \\
No. total abnormal blastocysts (\%) & $11(61.1)$ & $35(64.8)$ \\
\hline P:NS. & &
\end{tabular}

Table 3. Percentage of abnormalities for each chromosome analyzed in the vitrified and the fresh group.

\begin{tabular}{ccc}
\hline $\begin{array}{c}\text { Chromosome } \\
\text { analyzed }\end{array}$ & Vitrified Group & Fresh Group \\
\hline 13 & $34.8 \%$ & $36.2 \%$ \\
15 & $28.3 \%$ & $40.8 \%$ \\
16 & $21.7 \%$ & $27.7 \%$ \\
17 & $19.6 \%$ & $21.5 \%$ \\
18 & $28.3 \%$ & $29.2 \%$ \\
21 & $45.7 \%$ & $33.1 \%$ \\
22 & $19.6 \%$ & $31.5 \%$ \\
XY & $26.1 \%$ & $23.1 \%$ \\
\hline
\end{tabular}

$\mathrm{P}: \mathrm{NS}$.

significantly during the last decade [17-26,85-88].

Vitrification is the glass-like solidification of a solution at low temperature, without ice crystal formation. This is possible through the extreme elevation in viscosity during freezing. This can be achieved by increasing the freezing and warming rates and/or increasing the concentration of the cryoprotectants [89]. Unlike slow freezing, vitrification results in the total elimination of ice crystal formation, within the cells being vitrified and outside the cells in the surrounding solution [78]. Oocyte vitrification avoids meiotic spindle damage and has resulted in survival rates of over $80 \%$ [19-21,23,48,90]. Additionally, pronuclear zygotes [91], cleavage-stage embryos [82], and blastocysts [92-94] have been successfully vitrified.

During the present study has shown the feasibility and efficiency of oocyte vitrification. We have showed high recovery and post warm survival in vitrified oocytes, and similar fertilization rates and preimplantational development embryo with vitrified and fresh oocytes, results that confirm data showed previously by others investigators $[19,20,23]$. In the present study, the vitrification procedures were all carried out using the Cryolock device, which has a similar design and handle to those of Cryotop. The Cryolock has been designed and developed specifically for use in vitrification procedures with slots 
in the ends and top for better manipulation, avoiding the risk that these loosen or fall during their use or storage (Biodiseño-Colombia). Moreover, the vitrification procedure, cryoprotectants and warming media (Cecolfes, Colombia) and the Cryolock device (Biodiseño, Colombia) used during oocytes vitrification procedure has been showed previously to be efficient, safety and don't affect the viability and physiology of oocytes [20].

In recent years PGD technology has been increasingly used by infertile patients undergoing IVF treatment, in order to screen their embryos for chromosomal abnormalities. Aneuploidy is extremely common in human embryos and leads to developmental arrest, implantation failure and spontaneous abortion. The inadvertent transfer of chromosomally abnormal embryos is believed to explain a significant proportion of failed IVF cycles. By screening for aneuploidy and ensuring the transfer of chromosomally normal embryos it has been suggested that a variety of IVF outcomes (including implantation and pregnancy rates) can be improved.

Advances in vitrification methods have led to egg survival rate over $85 \%$ and the pregnancy rates comparable to those achieved with autogenous and donated fresh oocytes [17,19-21,23,25,26,48,89,95,96]. Additionally, has been demonstrated that vitrification of in-vitro maturated oocytes results in high survival rates, normal meiotic spindle and chromosome alignment and no increased incidence of aneuploidy compared to those cryopreserved by slow freezing [97]. However, no information is available about the chromosomal status of human embryos resulting from vitrification and warming procedure, fertilization and preimplantational development embryo.

In the present study, hasn't been observed deleterious effect of vitrification procedure on the oocyte viability from fertilization to blastocyst stage and principally the morphological characteristics of blastocyst at day 5, similar results to observed by García et al. [20].

On the other hand, has been showed similar aneuploidy rates in embryos at day $3(66.1 \%$ vs. $71.9 \%)$ and in those embryos that achieved the blastocyst stages (66.1\% vs. 64.8\%) from vitrified and fresh oocytes respectively. Similar results were obtained by Baart et al. [98] and Munné et al. [50] when analyzed 196 and 1800 embryos from oocyte donation cycles respectively. They observed that $66 \%$ and $57 \%$ of embryos were abnormal for 10 and 8 chromosomes analyzed including $\mathrm{X}, \mathrm{Y}, 1,7$, $13,15,16,18,21$ and 22. These results are direct evidence that oocytes conserve intact their capability of fertilization and potential the reach the blastocyst stage after being vitrified similar to that observed with fresh oocytes. This study shown that a correct repolymerization of the spindle may occur during the restoration of the physiological conditions after warming, leading to the right sequence of events involved in the completion of meiosis and fertilization., Finally, our results suggest that vitrification procedure has a lower impact on oocyte physiology, does not affect the normal preimplantational embryo development or increase the aneuploidies incidences compared to those observed in embryos from fresh oocytes. Additionally, Chian et al. [18] analyzed the obstetric and perinatal outcomes in 165 pregnancies and 200 infants conceived following oocytes vitrification cycles in three reproductive centers. The results indicate that the mean birth weight and incidence of congenital anomalies are comparable to that of spontaneous conceptions in fertile women or infertile women undergoing in-vitro fertilization treatment. These results confirm that oocytes conserve their capability of fertilization and potential to reach the blastocyst stage after being vitrified, similar to the data obtained with fresh oocytes.

On the other hand, the vitrification of human oocytes solves the legal restrictions and ethical problems [99] associated with the cryopreservation of embryos in patients undergoing in vitro fertilization procedures. Also, it may offer the possibility of extending the reproductive capability of young women with malignant diseases in cases where the treatment by surgery, chemotherapy or radiotherapy may compromise the ovarian reserve [100-103]. Moreover, it also provides alternatives for infertile patients who are subject to ovarian hyperstimulation syndrome, poor responder to ovarian stimulation, premature ovarian failure or who require oocyte donation [100].

In conclusion, our observations demonstrate that vitrification is an efficient procedure for oocyte cryopreservation, the developmental competence of vitrified MII oocytes to rich the blastocyst stage and the chromosomal status are similar to those observed with fresh oocytes. In conclusion, the vitrification procedure represents the best current choice for creation of reliable egg banks.

\section{REFERENCES}

[1] Chen, C. (1986) Pregnancy after human oocytes cryopreservation. Lancet, 1, 884-886. doi:10.1016/S0140-6736(86)90989-X

[2] Allan J. (2004) Pregnancy from intracytoplasmic injection of a frozen-thawed oocyte. The Australian and New Zealand Journal of Obstetrics and Gynaecology, 44, 588. doi:10.1111/j.1479-828X.2004.00317.x

[3] Levi Setti, P.E., Albani, E., Novara, P.V., Cesana, A. and Morreale, G. (2006) Cryopreservation of supernumerary oocytes in IVF/ ICSI cycles. Human Reproduction, 21, 370-375. doi:10.1093/humrep/dei347

[4] Nawroth, F. and Kissing, K. (1998) Pregnancy after intracytoplasmic sperm injection (ICSI) of cryopreserved human oocytes. Acta Obstet Gynecol Scand, 77, 462-463. 
[5] Porçu, E., Fabbri, R. and Seracchioli, R. (1997) Birth of a healthy female after intracytoplasmic sperm injection of cryopreserved human oocytes. Fertility and Sterility, 68, 724-726. doi:10.1016/S0015-0282(97)00268-9

[6] Van Uem, J.F., Siebzehnrubl, E.R., Schuh, B., Koch, R., Trotnow, S. and Lang, N. (1987) Birth after cryopreservation of unfertileized oocytes. Lancet, 1, 752-753. doi:10.1016/S0140-6736(87)90398-9

[7] Young, E., Kenny, A., et al. (1998) Triplet pregnancy after intracyto-plasmic sperm injection of cryopreserved oocytes: Case report. Fertility and Sterility, 70, 360-361. doi:10.1016/S0015-0282(98)00150-2

[8] Gook, D., Schiewe, M.C., et al. (1995) Intracytoplasmic sperm injection and embryo development of human oocytes cryopreserved using 1, 2-propanediol. Human Reproduction, 10, 2637-2641.

[9] Borini, A., Bonu, M.A., et al. (2004) Pregnancies and births after oocyte cryopreservation. Fertility and Sterility, 82, 601-605. doi:10.1016/j.fertnstert.2004.04.025

[10] Fabbri, R., Porçu, E., et al. (2001) Human oocytes cryopreservation: New perspectives regarding oocytes survival. Human Reproduction, 16, 411-416. doi:10.1093/humrep/16.3.411

[11] Porçu, E., Fabbri, R. and Damiano, G. (2000) Clinical o experience and applications of oocytes cryopreservation. Molecular and Cellular Endocrinology, 69, 33-37.

[12] Tucker, M.J., Morton, P.C., et al. (1998) Clinical application of human egg cryopreservation. Human Reproduction, 13, 3156-3159. doi:10.1093/humrep/13.11.3156

[13] Winslow, K.L., et al. (2001) Oocyte cryopreservation a three year follow-up of six- teen births. Fertility and Sterility, 76, 120. doi:10.1016/S0015-0282(01)02354-8

[14] Oktay, K., Cil, A.P. and Bang, H. (2006) Efficiency of oocyte cryopreservation: A meta-analysis. Fertility and Sterility, 86, 70-80. doi:10.1016/j.fertnstert.2006.03.017

[15] Al-Hasani, S., Butuhan, O. and Koutlaki, N. (2007) Three years of routine vitrification of human zygotes: Is it still fair to advocate slow-rate freezing? Reproductive BioMedicine Online, 14, 288-293. doi:10.1016/S1472-6483(10)60869-3

[16] Vajta, G. and Nagy, Z.P. (2006) Are programmable freezers still needed in the embryo laboratory? Review on vitrification. Reproductive BioMedicine Online, 12, 779796. doi:10.1016/S1472-6483(10)61091-7

[17] Antinori, M., Licata, E., et al. (2007) Cryotop vitrification of human oocytes results in high survival rate and healthy deliveries. Reproductive BioMedicine Online, 14, 72-79. doi:10.1016/S1472-6483(10)60766-3

[18] Chian, R.C., Huang, J.Y.H., et al. (2008) Obstetric and perinatal outcome in 200 infants conceived from vitrified oocytes. Reproductive BioMedicine Online, 16, 608-610. doi:10.1016/S1472-6483(10)60471-3

[19] Cobo, A., et al. (2008) Comparison of concomitant outcome achieved with fresh and cryopreserved donor oocytes vitrified by the Cryotop method. Fertility and Sterility, 89, 1657-1664. doi:10.1016/j.fertnstert.2007.05.050

[20] García, J.I., et al. (2011) Efficacy of oocyte vitrification combined with blastocyst stage transfer in an egg donation program. Human Reproduction, 26, 782-790. doi:10.1093/humrep/der008

[21] Kuwayama, M., et al. (2005) Highly efficient vitrifica- tion method for cryopreservation of human oocytes. Reproductive BioMedicine Online, 11, 300-308. doi:10.1016/S1472-6483(10)60837-1

[22] Kuwayama, M. (2007) Highly efficient vitrification for cryopreservation of human oocytes and embryos: Cryotop method. Theriogenology, 67, 73-80. doi:10.1016/j.theriogenology.2006.09.014

[23] Lucena, E., et al. (2006) Successful ongoing pregnancies after vitrification of oocytes. Fertility and Sterility, 85, 108-111. doi:10.1016/j.fertnstert.2005.09.013

[24] Nagy, Z.P., et al. (2009) Clinical evaluation of the efficacy of an oocyte donation program using egg cryo-banking. Fertility and Sterility, 92, 520-526. doi:10.1016/j.fertnstert.2008.06.005

[25] Selman, H., et al. (2006) Ongoing pregnancies after vitrification of human oocytes using a combined solution of ethylene glycol and dimethyl sulfoxide. Fertility and Sterility, 86, 997-1000.

doi:10.1016/j.fertnstert.2006.02.117

[26] Yoon, T.K., Lee, D.R. and Cha, S.K. (2007) Survival rate of human oocytes and pregnancy outcome after vitrification using slush nitrogen in assisted reproductive technologies. Fertility and Sterility, 88, 925-926. doi:10.1016/i.fertnstert.2006.12.071

[27] Gardner, D.K., et al. (2007) Analysis of oocyte physiology to improve cryopreservation procedures. Theriogenology, 67, 64-72. doi:10.1016/j.theriogenology.2006.09.012

[28] Larman, M.G., Sheehan, C.B. and Gardner, D.K. (2006) Vitrification of mouse pronuclear oocytes with no direct liquid nitrogen contact. Reproductive BioMedicine Online, 12, 66-69. doi:10.1016/S1472-6483(10)60982-0

[29] Jones, A., Van Blerkom, J., Davis, P. and Toledo, A. (2004) Cryopreservation of metaphase II human oocytes effects mitochondrial membrane potential: Implications for developmental competence. Human Reproduction, 19, 1861-1866. doi:10.1093/humrep/deh313

[30] Nottola, S.A., et al. (2007) Ultrastructure of human mature oocytes after slow cooling cryopreservation using different sucrose concentrations. Human Reproduction, 22, 1123-1133. doi:10.1093/humrep/del463

[31] Schatten, G., Simerly, C. and Schatten, H. (1985) Microtubule configuration during fertilization, mitosis, and early development in the mouse and the requirement for egg microtubule-mediated motility during mammalian fertilization. Proceedings of the National Academy of Sciences, 82, 4152-4156. doi:10.1073/pnas.82.12.4152

[32] Bianchi, V., et al. (2005) Meiotic spindle imaging in human oocytes frozen with a slow freezing procedure involving high sucrose concentration. Human Reproduction, 20, 1078-1083. doi:10.1093/humrep/deh736

[33] Chen, S.U., et al. (2000) Cryopreservation of mature human oocytes by vitrification with ethylene glycol in straws. Fertility and Sterility, 74, 804-808. doi:10.1016/S0015-0282(00)01516-8

[34] Chen, S.U., et al. (2001) Vitrification of mouse oocytes using closed pulled straws (CPS) achieves a high survival and preserves good patterns of meiotic spindles, compared with conventional straws, open pulled straws (OPS), and grids. Human Reprod, 16, 2350-2356.

[35] Chen, C.K., et al. (2004) Evaluation of meiotic spindles in thawed oocytes after vitrification using polarized light 
microscopy. Fertility and Sterility, 82, 666-672. doi:10.1016/j.fertnstert.2003.12.053

[36] Chen, S.U. and Yang, Y.S. (2009) Slow freezing or vitrification of oocytes: Their effects on survival and meiotic spindles, and the time schedule for clinical practice. Taiwanese Journal of Obstetrics and Gynecology, 48, 1522. doi:10.1016/S1028-4559(09)60030-9

[37] Coticchio, G., et al. (2005) Criteria to assess human oocyte quality after cryopreservation. Reproductive BioMedicine Online, 11, 421-427. doi:10.1016/S1472-6483(10)61133-9

[38] De Santis, L., et al. (2007) Objective evaluation of the viability of cryopreserved oocytes. Reprod Biomed Online, 15, 338-345. doi:10.1016/S1472-6483(10)60348-3

[39] Eroglu, A., et al. (1998) Alterations of the cytoskeleton and polyploidy induced by cryopreservation of metaphase II mouse oocytes. Fertility and Sterility, 69, 944957.

[40] Larman, M.G., et al. (2007) Maintenance of the meiotic spindle during vitrification in human and mouse oocytes. Reproductive BioMedicine Online, 15, 692-700. doi:10.1016/S1472-6483(10)60537-8

[41] Rienzi, L., et al. (2004) Polscope analysis of meiotic spindle changes in living metaphase II oocytes during the freezing and thawing procedures. Human Reproduction, 19, 655-659. doi:10.1093/humrep/deh101

[42] Magistrini, M. and Szollosi, D. (1980) Effects of cold and of isopropyl-N-phenylcarbamate on the second meiotic spindle of mouse oocytes. European Journal of Cell Biology, 22, 699-707.

[43] Sathananthan, A.H., et al. (1992) The effects of cooling mouse oocytes. Journal of Assisted Reproduction and Genetics, 9, 139-148. doi:10.1007/BF01203754

[44] Wang, W.H., et al. (2001) Limited recovery of meiotic spindle in living human oocytes after cooling-rewarming observed using polarized light microscopy. Human Reproduction, 16, 2374-2378.

[45] Sathananthan, A.H., et al. (1988) The effects of cooling human oocytes. Human Reproduction, 3, 968-977.

[46] Van der Elst, J., et al. (1988) Effect of 1,2-propanediol and di- methylsulphoxide on the meiotic spindle of the mouse oocyte. Human Reproduction, 3, 960-967.

[47] Cao, Y.X., et al. (2009) Comparison of survival and embryonic development in human oocytes cryopreserved by slowfreezing and vitrification. Fertility and Sterility, 92, 1306-1311. doi:10.1016/j.fertnstert.2008.08.069

[48] Katayama, P., et al. (2003) High survival rate of vitrified human oocytes results in clinical pregnancy. Fertility and Sterility, 80, 223-224. doi:10.1016/S0015-0282(03)00551-X

[49] Kuwayama, M. (2001) Vitrification of human oocytes and embryos. In: Suzuki, S. Ed., IVF Update, Medical View Co., Tokyo, 230-234.

[50] Munné, S., et al. (2006) Wide range of chromosome abnormalities in the embryos of young egg donors. Reprod Biomed Online, 12, 340-346. doi:10.1016/S1472-6483(10)61007-3

[51] Silber, S., et al. (2003) Chromosomal abnormalities in embryos derived from TESE. Fertility and Sterility, 79, 30-38. doi:10.1016/S0015-0282(02)04407-2

[52] Coticchio, G., et al. (2009) Vitrification may increase the rate chromosome misalignment in the metaphase II spindle of human mature oocytes. Reproductive BioMedicine Online, 19, 29-34. doi:10.1016/S1472-6483(10)60281-7

[53] Martínez-Burgos, M., et al. (2011) Vitrification versus slow freezing of oocytes: effects on morphologic appearance, meiotic spindle configuration, and DNA damage. Fertility and Sterility, 95, 374-377. doi:10.1016/j.fertnstert.2010.07.1089

[54] Ciotti, P.M., et al. (2009) Meiotic spindle recovery is faster in vitrification of human oocytes compared to slow freezing. Fertility and Sterility, 91, 2399-2407. doi:10.1016/j.fertnstert.2008.03.013

[55] Hardy, K., et al. (1990) Human preimplantation development in vitro is not adversely affected by biopsy at the 8-cell stage. Human Reproduction, 5,708-714.

[56] Gianaroli, L., et al. (1999) Preimplantation diagnosis for aneuploidies in patients undergoing in vitro fertilization with poor prognosis: Identification of the categories for which it should be proposed. Fertility and Sterility, 72, 837-844. doi:10.1016/S0015-0282(99)00377-5

[57] Munné, S., et al. (2003) Improved implantation after Preimplantation genetic of aneuploidy. Reproductive BioMedicine Online, 7, 91-97. doi:10.1016/S1472-6483(10)61735-X

[58] Munné, S., et al. (2004b) Increased rate of aneuploid embryos in young women with previous aneuploid conceptions. Prenatal Diagnosis, 24, 638-647. doi:10.1002/pd.957

[59] Gianaroli, L., et al. (2007) Oocyte aneuploidy, pronuclear zygote morphology and embryo chromosomal complement. Human Reproduction, 22, 241-249. doi:10.1093/humrep/del334

[60] Magli, M.C., et al. (2001) Double locus analysis of chromosome 21 for Preimplantation genetic diagnosis of aneuploidy. Prenatal Diagnosis, 21, 1080-1085. doi: $10.1002 / \mathrm{pd} .248$

[61] Márquez, C., et al. (2000) Chromosome abnormalities in 1255 cleavage-stage human embryos. Reproductive BioMedicine Online, 1, 17-27. doi:10.1016/S1472-6483(10)61988-8

[62] Munné, S., et al. (2004a) Differences in chromosome susceptibility to aneuploidy and survival to first trimester. Reproductive BioMedicine Online, 8, 81-90. doi:10.1016/S1472-6483(10)60501-9

[63] Rubio, C., et al. (2003) Chromosomal abnormalities and embryo development in recurrent miscarriage couples. Human Reproduction, 18, 182-188. doi:10.1093/humrep/deg015

[64] Rubio, C., et al. (2005) FISH screening of aneuploidies in preimplantation embryos to improve IVF outcome. Reproductive BioMedicine Online, 11, 497-506. doi:10.1016/S1472-6483(10)61146-7

[65] Kahraman, S., et al. (2000) Healthy births and ongoing pregnancies by preimplantation genetic diagnosis in patients with advanced maternal age and recurrent implantation failure. Human Reproduction, 15, 2003-2007. doi:10.1093/humrep/15.9.2003

[66] Munné, S., et al. (1995) Embryo morphology, developmental rates, and maternal age are correlated with chromosomal abnormalities. Fertility and Sterility, 64, 382391.

[67] Munné, S., et al. (2002) Preimplantation genetic diagno- 
sis for advanced maternal age and other indications. Fertility and Sterility, 78, 234-236. doi:10.1016/S0015-0282(02)03239-9

[68] Pehlivan, T., et al. (2003) Impact of preimplantation genetic diagnosis on IVF outcome in implanttation failure patients. Reproductive BioMedicine Online, 6, 232237. doi:10.1016/S1472-6483(10)61715-4

[69] Wilding, M., et al. (2004) Preimplantation genetic diagnosis for treatment of failed in vitro fertilization embryo transfer and habitual abortion. Fertility and Sterility, 81, 1302-1307. doi:10.1016/j.fertnstert.2003.10.028

[70] Pellicer, A., et al. (1999) In vitro fertilization plus preimplantation genetic diagnosis in patients with recurrent miscarriage: an analysis of chromosome abnormalities in human preimplantation embryos. Fertility and Sterility, 71, 1033-1039. doi:10.1016/S0015-0282(99)00143-0

[71] Simón, C., et al. (1998) Increased chromosome abnormalities in human Preimplantation embryo after in vitro fertilization in patients with recurrent miscarriage. Reproduction, Fertility and Development, 10, 87-92. doi:10.1071/R98030

[72] Vidal, F., et al. (1998) FISH preimplantation diagnosis of chromosome aneuploidy in recurrent pregnancy wastage. Journal of Assisted Reproduction and Genetics, 15, 309312. doi:10.1023/A:1022552713015

[73] Werlin, L., et al. (2003) Preimplantation genetic diagnosis as both a therapeutic and diagnosis tool in assisted reproductive technology. Fertility and Sterility, 80, 467468. doi:10.1016/S0015-0282(03)00605-8

[74] Munné, S., et al. (2007) Maternal age, morphology, development and chromosome abnormalities in over 6000 cleavage-stage embryo. Reproductive BioMedicine Online, 14 628-634. doi:10.1016/S1472-6483(10)61057-7

[75] Reis Soares, S., et al. (2003) High frequency of chromosomal abnormalities in embryo obtained from oocyte donation cycle. Fertility and Sterility, 80, 656-657. doi:10.1016/S0015-0282(03)00787-8

[76] Cobo, A., et al. (2001) Use of fluorescence in situ hybridization to assess the chromosomal status of embryos obtained from cryopreserved oocytes. Fertility and Sterility, 75, 354-360. doi:10.1016/S0015-0282(00)01725-8

[77] Kuleshova LL, Lopata A. (2002) Vitrification can be more fa- vorable than slow cooling. Fertility and Sterility,; 78, 449-454. doi:10.1016/S0015-0282(02)03305-8

[78] Rall, W.F. (1987) Factors affecting the survival of mouse em- bryos cryopreserved by vitrification. Cryobiology, 24, 387-402. doi:10.1016/0011-2240(87)90042-3

[79] Velilla, E., Escudero, T. and Munné, S. (2002) Blastomere fixation technique and risk of misdiagnosis for PGD of aneuploidy. Reproductive BioMedicine Online, 4 210- 217. doi:10.1016/S1472-6483(10)61808-1

[80] Munné, S., et al. (1998) Preimplantation diagnosis of the aneuploidies most commonly found in spontaneous abortions and live births: X, 13, 14, 15, 16, 18, 21, 22. Pregnancy Diagnosis, 18, 1459-1466. doi:10.1002/(SICI)1097-0223(199812)18:13<1459::AID -PD514>3.0.CO;2-V

[81] Colls, P., et al. (2004) PGD analysis for aneuploidy in a patient heterozygous for a polymorphism of chromosome 16 (16qh-). Prenatal Diagnosis, 24, 741-744. doi:10.1002/pd. 887
[82] El-Danasouri, I. and Selman, H. (2001) Successful pregnancies and deliveries after a simple vitrification protocol for day 3 human embryos. Fertility and Sterility, 76, 400402. doi:10.1016/S0015-0282(01)01907-0

[83] Hong, S.W., et al. (1999) Improved human oocytes development after vitrification: A comparison of thawing methods. Fertility and Sterility, 72, 142-146. doi:10.1016/S0015-0282(99)00199-5

[84] Yoon, T.K., et al. (2000) Pregnancy and delivery of healthy infants developed from vitrified oocytes in a stimulated in vitro fertilization-embryo transfer program. Fertility and Sterility, 74, 180-181. doi:10.1016/S0015-0282(00)00572-0

[85] Kim, T.J., et al. (2010) Vitrification of oocytes procedures high pregnancy rates when carried out in fertile women. Fertility and Sterility, 93, 467-474. doi:10.1016/j.fertnstert.2008.12.094

[86] Rienzi, L., et al. (2010) Embryo development of fresh "versus" vitrified metaphase II oocytes after ICSI: A prospective randomized sibling-oocyte study. Reproductive BioMedicine Online, 25, 66-73.

[87] Sher, G., et al. (2008) Selective vitrification of euploid oocytes markedly improves survival, fertilization and pregnancy-generating potential. Reproductive BioMedicine Online, 17, 524-529. doi:10.1016/S1472-6483(10)60240-4

[88] Ubaldi, F., et al. (2010) Cumulative ongoing pregnancy rate achieved with oocyte vitrification and cleavage stage transfer without embryo selection in a standard infertility program. Human Reproduction, 25, 1199-1205. doi:10.1093/humrep/deq046

[89] Fahy, G.M., et al. (1984) Vitrification as an approach to cryopreservation. Cryobiology, 21, 407-426. doi:10.1016/0011-2240(84)90079-8

[90] Chian, R.C., et al. (2005) High survival rates and pregnancies of human oocytes following vitrification: Preliminary report. Fertility and Sterility, 80, 36. doi:10.1016/j.fertnstert.2005.07.086

[91] Isachenko, V., et al. (2005) Aseptic technology of vitrification of human pronuclear oocytes using open-pulled straws. Human Reproduction, 20, 492-496. doi:10.1093/humrep/deh605

[92] Lane, M., et al. (1999) Containerless vitrification of mammalian oocytes and embryos. Nat Biotechnol, 17, 1232-1236. doi:10.1038/70795

[93] Lieberman, J., et al. (2002) Blastocyst development after vitrification of multipronuclear zygotes using the flexipet denuding pipette. Reproductive BioMedicine Online, $\mathbf{4}$, 146-150. doi:10.1016/S1472-6483(10)61932-3

[94] Mukaida, T., et al. (2003) Vitrification of human blastocysts using cryoloops: Clinical outcome of 223 cycles. Human Reproduction, 18, 384-391. doi:10.1093/humrep/deg047

[95] Cobo, A., et al. (2010) Use of cryo-banked oocytes in an ovum donation programme: A prospective, randomized, controlled, clinical trial. Human Reproduction, 25, 22392246. doi:10.1093/humrep/deq146

[96] Yoon, T.K., et al. (2003) Live births after vitrification of oocytes in a stimulated in vitro fertilization-embryo transfer program. Fertility and Sterility, 79, 1323-1326. doi:10.1016/S0015-0282(03)00258-9

[97] Huang, J.Y., et al. (2007) Comparison of spindle and 
chromosome configuration in vitro and in vivo matured mouse oocytes after vitrification. Fertility and Sterility, 90, 1424-1432. doi:10.1016/j.fertnstert.2007.07.1335

[98] Baart, E.B., et al. (2006) Preimplantation genetic screening reveals a high incidence of aneuploidy and mosaicism in embryos from young women undergoing IVF. Human Reproduction, 21, 223-233. doi:10.1093/humrep/dei291

[99] Parmegiani, L., et al. (2008) Freezing within $2 \mathrm{~h}$ from oocyte retrieval increases the efficiency of human oocyte cryopreservation when using slow freezing/rapid thawing protocol with high sucrose concentration. Human Reproduction, 23, 1771-1777. doi:10.1093/humrep/den119

[100] Gidoni, Y., et al. (2008) Fertility presservation in patients with non-oncological conditions. Reproductive BioMedi- cine Online, 16, 792-800. doi:10.1016/S1472-6483(10)60144-7

[101] Li, X.H., et al. (2005) Cryopreserved oocytes of infertile couples undergoing assisted reproductive technology could be an important source of oocyte donation: A clinical report of successful pregnancies. Human Reproduction, 20, 3390-3394. doi:10.1093/humrep/dei262

[102] Porçu, E., et al. (2008) Healthy twins delivered after oocyte cryopreservation and bilateral ovariectomy for ovarian cancer. Reproductive BioMedicine Online, 17, 265-267.

[103] Yang, D., et al. (2007) Live birth after the transfer of human embryos developed from cryopreserved oocytes harvested before cancer treatment. Fertility and Sterility, 87, 4. 